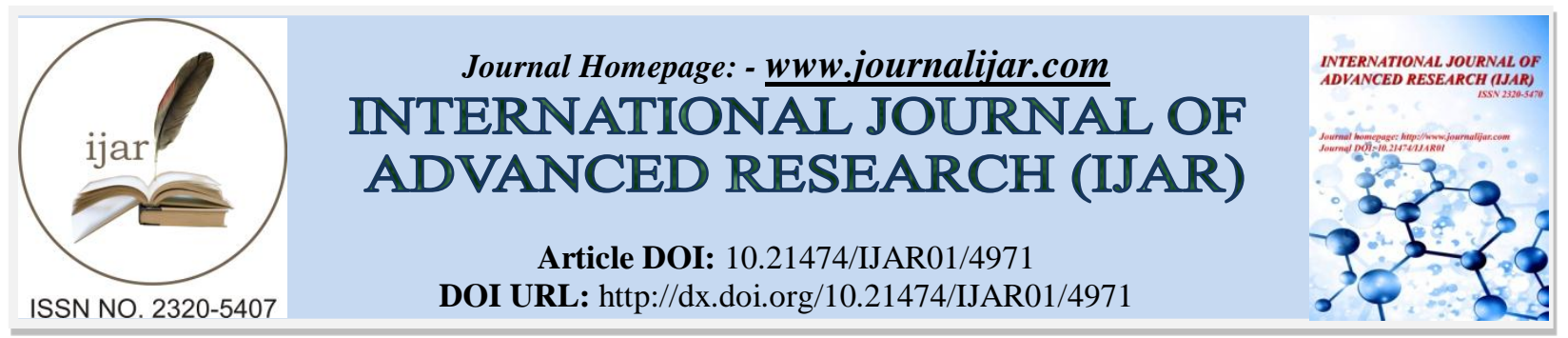

RESEARCH ARTICLE

\title{
EFFECT OF NEUROMUSCULAR ELECTRICAL STIMULATION ON WRIST EXTENSORS TO IMPROVE HAND FUNCTION IN CHILDREN WITH SPASTIC CEREBRAL PALSY.
}

1. MPT (Pediatric)

Nibedita Samal ${ }^{1}$, Mrs. Monalisa Pattnaik ${ }^{2}$ and Dr Patitapaban Mohanty ${ }^{3}$.

2. Assistant professor, Department of Physiotherapy, SVNIRTAR, Cuttack, Odisha.

3. Associate Professor \& HOD, Department of Physiotherapy, SVNIRTAR, Cuttack, Odisha.

\section{Manuscript Info}

Manuscript History

Received: 27 May 2017

Final Accepted: 29 June 2017

Published: July 2017

Key words:-

CP-cerebral palsy, NMES-

neuromuscular electrical stimulation,

QUEST- Quality of upper extremity skill

test, MMAS- modified modified

asworth's scale, GMFCS level- Gross

motor function classification system

Level, MACS- Manual ability

classification system.

\section{Abstract}

Introduction: Impairments in the upper extremity in CP may include muscle tone abnormality, imbalance between agonist and antagonist group of muscles, spasticity, alignment problem, decreased strength and impaired motor control. The combination of these primary impairments has been targeted for various rehabilitation strategies in Physical therapy. Aim of the study is to find out the effect of neuromuscular electrical stimulation on wrist extensors to improve hand function in spastic cerebral palsy children.

Methodology: The selected subjects were randomly assigned in to experimental and control groups with 15 subjects each. Experimental group received task oriented exercises along with neuromuscular electrical stimulation on wrist extensors for 6 weeks. The control group received only task oriented exercises. Total duration of treatment is 30 minutes per day given 5 days in a week for 6 weeks.

Results: Result of the study showed that both the control and experimental group improved their hand function. However the experimental group showed better changes as compared to the control group.

Conclusion: The study demonstrated that the combined effect of neuromuscular electrical stimulation along with task oriented exercises are beneficial for improving hand function in spastic cerebral palsy children.

Copy Right, IJAR, 2017,. All rights reserved.

\section{Introduction:-}

Cerebral palsy (CP) describes a group of permanent disorders of the development of movement and posture, causing activity limitation, that are attributed to non-progressive disturbances that occurred in the developing fetal or infant brain. The motor disorder of cerebral palsy is often accompanied by disturbances of sensation, perception, cognition, communication, behavior, epilepsy, and secondary musculoskeletal problems.[1] Motor deficits in CP include features like weakness, fatigue, in coordination, spasticity, clonus, rigidity, and spasms. Spasticity can lead to muscle stiffness, functional impairment, and atrophy. If not treated, it can progress to muscle fibrosis, contractures, and subsequent musculoskeletal deformities.[2] The hand is typically affected, to the detriment of the performance of activities of daily living. The stereotypical posture of the wrist flexion and ulnar deviation coupled with finger and thumb flexion into the palm hinders grasp and release. Impairments in the upper extremity in CP may include 
muscle tone abnormality, imbalance between agonist and antagonist group of muscles, spasticity and alignment problem, decreased strength and impaired motor control. The combination of these primary impairments has been targeted for various rehabilitation strategies.[3] Researchers showed that spasticity leads to generalized muscle fiber atrophy with predominance of type 1 fibers. The non spastic antagonist muscle is over lengthened and weak. This atrophy contributes to fatigue and limited function and muscle imbalance around the joints in CP children.[4] Neuromuscular electrical stimulation (NMES) is the application of electrical current either transcutaniously or percutaneously to innervated superficial or deep muscles respectively by exciting the intact efferent peripheral nerve to stimulate muscle fibers, augments muscle contraction, increase range of motion and increase sensory awareness.[5] NMES is thought to reduce spasticity through stimulation of the antagonist muscle, reduce co contraction and /or create soft tissue changes and permit an increase range of motion. It also enhances the strength. So it provides an alternative to resistive exercise technique for children with poor selective motor control.[6] Primary physiologic mechanism for the initial gain in strength is improvement in the ability to recruit motor units. So NMES may also help to increase the patient's volitional strength through learned recruitment of motor units. Over time the muscle will adapt in response to specific force generating demands placed on it like muscle hypertrophy due to increase in the contractile protein.[7] When NMES is added with task oriented movements with several repetitions motor learning takes place. Motor learning theory asserts that motor learning to occur, activities must be repetitive and task or goal oriented and at the limit of performance .[8]

\section{Aim Of The Study:-}

To compare the effectiveness of neuromuscular electrical stimulation along with task oriented Physiotherapy treatment with only task oriented Physiotherapy treatment for improvement of hand function in spastic cerebral palsy.

\section{Methodology:-}

30 spastic cerebral palsy children were selected from the Pediatric Physiotherapy Department of SVNIRTAR, fulfilling the inclusion criteria were randomly allotted in two groups. All subjects had undergone an initial baseline assessment of QUEST and MMAS. GMFCS level- Gross motor function classification system Level, MACS level Manual ability classification systems were assessed in order to exclude more dependent group of cerebral palsy children.

Inclusion criteria:- Children diagnosed with spastic cerebral palsy, Age of the children vary between 3-10 years, they should seat independently and follow the command of the therapist, Both the genders included in the study, Spasticity within grade 2 in MMAS, GMFCS level I, II, III, MACS level I, II, III.

Exclusion criteria:- Children with mental retardation, Children with epileptic attack in past 6 months, Children with Athetoid, ataxic, flaccid cerebral palsy, Children with congenital deformity of limbs, spine and neck, Children with visual or auditory deficit.

\section{Procedure:-}

All subjects after meeting the inclusion and exclusion criteria were randomly divided into two groups, experimental and control groups. Consent forms were filled up by their parents. Participants underwent an initial baseline assessment of QUEST, MMAS. The Quality of Upper Extremity Skills Test is an outcome measure designed to evaluate movement patterns and hand function in children with cerebral palsy. The purpose of the QUEST is to evaluate quality of upper extremity function in four domains: dissociated movement, grasp, protective extension, and weight bearing. Test retest reliability of QUEST and its domains ranges from 0.75 to 0.95.[9] The Modified Ashworth Scale (MAS) is the most widely used clinical test for the measurement of muscle spasticity. The modified modified asworth's scale (MMAS) had good inter-rater reliability $(\mathrm{K}=0.63, \mathrm{SE}=0.11, \mathrm{p}<0.001)$ for the assessment of wrist flexors spasticity in hemiplegic patients.[10] All the two groups received task oriented physiotherapy exercises which includes grasping and releasing 1 inch size wooden cubes using thumb and palm, grasping a cereal, grasping a crayon or pencil and drawing different shapes, reaching for toys placed forward, backward and sideways. Doing peg board activities, reaching for a football using both upper limb and then perform throwing the ball, holding one small glass or water bottle and perform drinking activity etc. The exercises were designed to improve both unimanual and bimanual hand dexterity. These exercises repeated minimum 10 times and given 5 days/week for 6 weeks in the department and also advised to repeat the same exercises at home with same repetitions. The experimental group in addition received NMES by Enraf Nonius muscle stimulator. The NMES protocol: Symmetric biphasic pulses. Pulse duration 280 micro seconds (Kamper et al) frequency was pre set by the 
machine, ramp on / off time was 2:1. The intervention period was 30 minutes per day, 5 days per week for 6 weeks. After completion of 6 weeks all participants received follow up assessments.

\section{Data Collection:-}

Pretest QUEST and MMAS scores were taken prior to the intervention and post test measurements were taken after completion of 6 weeks of intervention.

\section{Data Analysis:-}

Data was analyzed using non parametric Mann Whitney U test to know the between group difference in change score. The change score was taken as post score minus pre score for both the groups. Wilcoxon Signed Rank test was used to know the difference within the groups, 0.05 level of significance was used for hypothesis testing. Analysis was performed using SPSS versions 23 package.

\section{Results:-}

The overall result of the study showed significant improvement of hand function measured by QUEST score in both the groups. Experimental group showed better improvement than control group. The graph 1 shows the change in QUEST. The change is more in the experimental group than the control group. Mann Whitney U test shows Z score -3.218 , value of test is 36.000 , with $p<0.001$. This indicates that there is significant difference in change in scores. The graph 2 shows the change in MMAS. Mann Whitney U test shows Z score -1.795 , value of test is 90.000 , with $\mathrm{p}<0.367$. This indicates no significant changes in the score.

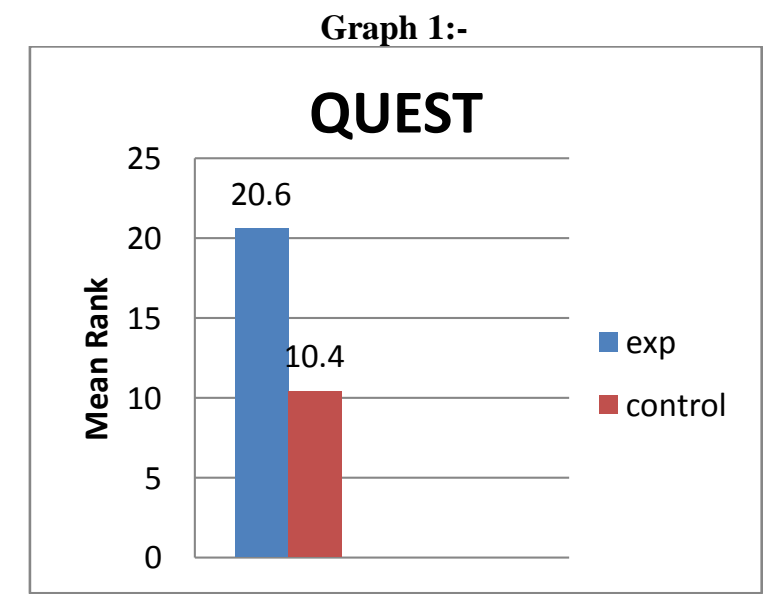

Graph 1:- indicates the change in Quest score between experimental and control groups by Mann Whitney U test. It shows the mean rank experimental group is 20.6 and control group is 10.4 .

Graph 2:

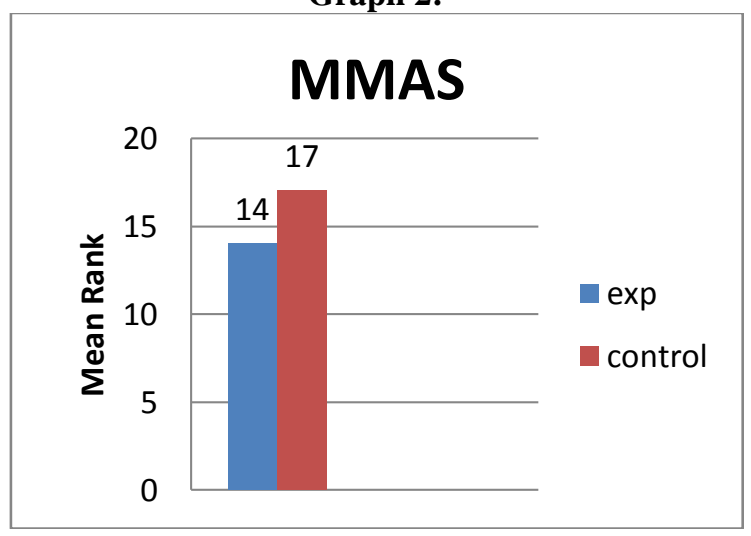

Graph 2:- indicates the change of MMAS score between experimental and control group by Mann Whitney U test. The mean rank of experimental group is 14 and control group is 17. 


\section{Discussion:-}

Overall results of the study showed, significant improvement in hand function in both experimental and control group in QUEST score, after six weeks of treatment protocol. However the improvement was significantly more in experimental group than control group. But the spasticity component which was measured by MMAS, not improved significantly in both the groups.

\section{Quest:-}

The QUEST score was used to evaluate the hand function in spastic cerebral palsy children in this study. Only two component of QUEST i.e.; grasp and dissociated movements of bilateral upper extremity were evaluated. Mann Whitney $U$ test shows significant improvement between the two groups with $\mathrm{p}<0.001$, Mean rank 20.6 in experimental group and 10.4 in control group. In experimental group, the $\%$ of improvement in hand function was $11.97 \%$ and in control group, it was $2.04 \%$.

Both the groups received task oriented exercises programs. The exercises were designed to improve both unimanual and bimanual hand dexterity. Each exercise was repeated minimum 10 times and given 5 days/week for 6 weeks in the department and also advised to repeat the same exercises at home with same repetitions. That means each exercises were repeated 20 times per day. So in a week each exercises were repeated 100 times and in six weeks 600 repetitions. There were 10 components of exercise designed for the present study. So overall 6000 repetition completed at the end of $6^{\text {th }}$ week. So this much repetition could help in creating motor learning in both the groups. Motor learning theory suggests that, procedural learning develops slowly through repetitions of an act over many trials, and it is expressed through improved performance of the task it was practiced.[11] Significant improvement in control group which received task oriented exercises alone could also be due to improved motor control and increased strength of upper limb.[12] Task oriented exercise has also been linked to improve cortical reorganization. Animal studies can demonstrated that task oriented training can restore function by using non affected part of the brain which are generally adjacent to the lesion [13] and/or recruiting the supplementary areas of brain.[14] Neural plastic changes have also been demonstrated in the human brain following stroke and task specific intervention.[1517] Jang S H et al. also noted decrease in the unaffected and increase in the affected primary sensory motor cortex activities along with functional recovery in stroke patients who received task oriented exercises.[18] Classen et al. using focal transcranial magnetic stimulation, has shown that task oriented exercises in comparison to traditional stroke rehabilitation, yields long lasting cortical reorganization specific to the area being used.[19] Most researchers recommended that the more the task is practiced better the overall performance. According to the Kilgard and Merzenich repetition play a major role in inducing and maintaining brain changes.[20] Task oriented exercise session consisting of hand and thumb movements, for as short as 15 minutes have resulted in lasting cortical representational changes.[21] Also research has demonstrated that rehabilitation may be more successful if the task and stimuli are important and meaningful to the person.[22] So in this research the task specific training are designed as per the age and requirement of the CP child. Salem et al. supported the efficacy of task-oriented strength training for improving mobility function in children with cerebral palsy.[23] Chiang soon song evaluated the effect of task-oriented arm approach on functional activities of the affected arm in children with CP and found task oriented exercises are beneficial.[24]

\section{The underlying mechanism of hand function training:}

The more practice and repetition are key components of training which lead to more sensory input, feedback and permanent changes as new strategies and motor plan produced lead to learning a new skill or restore the lost skill. The nervous system provide the:

1. Sensory processing for perception of body orientation in space provided by visual, vestibular, and somatosensory systems

2. Sensory-motor integration essential for linking sensation to motor responses (centrally programmed postural adjustments that precede voluntary movement)..

3. Mechanism of new motor strategy:

Which mean learning of new skill then formation of motor command via tracts to final common pathway (alpha and gamma $\mathrm{MN}$ ) to perform new behavior of skills or reacquisition of skills. Despite the development of indices designed to assess the function of the hand's grip strength assessment remains the cornerstone of most longitudinal studies designed to show functional change in the hand. Experimental group improved more than the control group. The additional improvement in this group could be due to the effect of NMES. 
NMES elicit muscle contraction by two different mechanisms. First, the overload principle, resulting in greater muscle strength by increasing the cross-sectional area of the muscle, and second, selective recruitment of type 11 muscle fibers i.e. fast twitch, large diameter fibers causing improved synaptic efficiency of the muscle, which lead to improvement in muscle strength.[25] The central deficit contributes to weakness in cerebral palsy. The findings suggest that muscle spasticity and weakness coexist in $\mathrm{CP}$ that result, inability of the damaged motor pathways to provide sufficient excitatory drive to the motoneuron pool to fully activate all available motor units. The NMES loaded the musculotendinous unit to a greater degree \& higher loading may have exceeded the threshold required to induce a training response. Stimulating the antagonist to the spastic muscle activates the 1a afferent of the antagonist, which activate the 1a interneuron and reciprocally inhibit the spastic (agonist) alpha motor neuron of the muscle. The electrical stimulation therapy in the limb's muscle has been tried in patients with upper motor neuron lesion as well as in patients with cerebral palsy. The results of the studies indicated that electrical simulation might be an effective tool for improving muscle strength.

NMES also facilitate motor learning. For children with cerebral palsy, where electrical stimulation may enhance muscle contraction and provide sensation so that a child can add a weak response with effective results.[26] So the additional improvement of the experimental group was due to improvement of strength and facilitation of motor learning through electrical stimulation.

Wright and Granat reported on the therapeutic effect of the FES (functional electrical stimulation) of the upper limb of eight children with CP. Cyclic FES was applied to the wrist extensor for 6 weeks. Improvement was observed in hand function and active wrist extension. Improvements were largely maintained until the end of the follow up period. The improvement in active wrist extension suggest that exercise of the wrist extensor with FES increase the strength of the muscle and therefore the range of motion, where the soft tissue around the joint permits further movement.[27]

M L van der Linden conducted a research on cerebral palsy children on improvement of hip extensor strength by electrical stimulation and he found electrical stimulation of the gluteus maximus significantly improved the hip extensor strength in children with cerebral palsy (CP).[28]

Amir El fiky conducted a systematic review to summarize the effect of the surface electrical stimulation in treatment of problem that affect the motor performance of the children with cerebral palsy. It has a significant result in improve gait, gross motor function in lower limb and upper limb and to improve trunk control and sitting balance, there is more evidence to use motor level than sensory level of stimulation and in motor the evidence to use NMES more than FES.[29]

Vaz DV et al. conducted an experimental study where he given strength training aided with electrical stimulation on wrist extensors. Significant strength gains were observed at 30 degree of flexion for flexors but no changes seen in passive stiffness of the muscle.[30]

In the present study it was found, no significant reduction of Spasticity in both the groups. But in the experimental group there was only $20 \%$ children showed mild reduction of spasticity.

\section{Mmas:-}

In the present study it was found, no significant reduction of Spasticity in both the groups, $p<0.367$ Mean rank of change in MMAS score of experimental group is 14 and control group is 17. In the experimental group there was only $20 \%$ children showed mild reduction of spasticity.

There is no significant reduction of the spasticity in both the groups. The pre test assessment done for both the groups. In the experimental group, there were total 15 children, among them 10 children were assessed with spasticity grade II and 5 with grade I in MMAS. And in control group out of 15 children, 13 children were having spasticity grade II and only 2 children with spasticity grade I. after the intervention post test assessment showed only $20 \%$ children of Experimental group were changed from grade II to grade I. but there were no change of spasticity seen in control group. This small change of spasticity could be due to small sample size and short duration of intervention used in this study.

Some of the studies reporting the effect of electrical stimulation on upper extremity spasticity are limited. 
Baker et al. reported an increase in wrist and finger extension in 16 adult patients with hemiplegia with unilateral flexor spasticity. However patients seen at 1 and 2 months after cessation of electrical stimulation had developed increased flexor contracture despite attempting to maintain ROM by passive exercises and splint.[31]

Kamper et al. reported on the effects of NMES treatment with respect to potential impairment mechanisms. This pilot study evaluated eight patients with CP who received 3 months of NMES targeting wrist flexor and extensor muscles. Differences in spasticity and passive resistance were not significant. The authors concluded that the observed increase in isometric wrist-extension torque likely arise from reduced flexor co-activation.[32]

\section{Conclusion:-}

The combined effect of task oriented exercises with NMES is more beneficial than only task oriented exercises for hand function improvement in patient with spastic cerebral palsy.

\section{Limitations:-}

Small sample size, short duration study, no follow up was done after completion of the treatment and outcome measures for strength improvement were not taken.

\section{Clinical Utility:-}

As the study showed significant improvement of hand function by the combined effect of task oriented exercises and NMES, so this combination of treatment can be incorporated in physiotherapy practices for patients with hand function impairment.

\section{References:-}

1. Peter Rosenbaum et.al. (2007) A report: the definition and classification of cerebral palsy.

2. MMS Jan et al. (2006) Cerebral Palsy: Comprehensive Review and Update, 26(2): 123 - 132.

3. Kamper DG et al. (2006) Effect of neuromuscular electrical stimulation treatment of cerebral palsy on potential impairement mechanisms a pilot study. Pediatr Phys Ther. 18(1): $31-8$.

4. Pepe KE (1997) Therapeutic electrical stimulation for the treatment of disuse muscle atrophy in cerebral palsy, pediatric physiotherapy 9: $110-12$.

5. Cramic J (1993) Clinical use of Neuromuscular electrival stimulation for children with cp. Part 1, lower extremity physical therapy $73: 505-513$.

6. Fiky AE (2012) Efficacy of surface electrical stimulation in treatment of CP children: A systematic review. Cario university. 17(1).

7. Reed B (1997) The physiology of NMES, pediatric physiotherapy 9: $96-102$.

8. Schmidt et al (1999) Motor control and learning a behavioral emphasis. 3rd edition champagne IL human kinetic publisher $261-285$.

9. Matteo CD et al. (1992) Quality of Upper Extrimity Skills Test. QUEST Manual.

10. Naghadi S et al. (2007) A preliminary study into the criterion validity of the Modified Modified Ashworth Scale using the new measure of the alpha motoneuron excitability in spastic hemiplegia. Electromyography and Neurophysiology. 47(3): $187-192$.

11. Cook AS, Woollacott MH (2001) Motor Control: Theory and Practical Applications. Lippincott Williams \& Wilkins. 2nd edition.

12. Birkenmeier RL, Prager EM, Lang CE (2010) Translating animal doses of task specific training to people with chronic stroke in 1-hour therapy sessions: a proof concept study. Neurorehabilitation and neural repair 24(7): $620-635$.

13. Nudo RJ, Milliken GW (1996) Reorganization of movement representations in primary motor cortex following focal ischemic infarcts in adult squirrel monkeys. Journal of Neurophysiology 75: $2144-2149$.

14. Plautz EJ, Milliken GW, Nudo RJ (2000) Effects of repetitive motor training on movement representations in adult squirrel monkeys: role of use versus learning. Neurobiology of learning and memory 74(1): $27-55$.

15. Richards LG et al. (2008) Movement-dependent stroke recovery a systematic review and Meta analysis of TMS and fMRI evidence. Neuropsychology 46(1): 3 .

16. Calautti $C$ et al. (2001) Sequential activation brain mapping after subcortical stroke: changes in hemispheric balance and recovery. Neuroreport 12: $3883-3886$.

17. Carey LM, Seitz RJ (2007) Functional neuroimaging in stroke recovery and neurorehabilitation: conceptual issues and perspectives. International Journal of Stroke 2: 245 - 264. 
18. Jang SH et al. (2003) Cortical reorganization induced by task-oriented training in chronic hemiplegic stroke patients.Neuroreport 14(1): $137-141$.

19. Classen J et al. (1998) Rapid plasticity of human cortical movement representation induced by practice. J Neurophysiol 79(2): $1117-1123$.

20. Kilgard MP, Merzenich MM (1998) Cortical map reorganization enabled by nucleus basalis activity. Science 279(5357): $1714-1718$

21. Duncan PW, Badke MB (1987) Stroke Rehabilitation. The Recovery of Motor Function. Chicago: Year Book.

22. Byl N et al. (2003) Effectiveness of Sensory and Motor Upper Limb Following The Principles of Neuro plasticity: Patients Stable Post stroke Rehabilitation of The Neuro rehabilitation And Neural Repair 17(3): 176 191.

23. Salem Y, Godwin EM (2009) Effects of task-oriented training on mobility function in children with cerebral palsy. Neurorehabilitation. 24(4): $307-13$.

24. Song CS (2014) Effects of Task-oriented Approach on Affected Arm Function in Children with Spastic Hemiplegia Due to Cerebral Palsy J Phys Ther Sci. 26(6): 797 - 800.

25. Delitto A, Mackler LS (1990) The two theories of muscle strength augmentation using percutaneous electrical stimulation. Phys the 70: $158-64$.

26. Robertson V et al. (2006) Electrotherapy explained principles and practice. 3rd edition. Electrical stimulation of nerve and muscle. pg no 82.

27. Wright PA, Granat MH (2000) Therapeutic effects of functional electrical stimulation of the upper limb of eight children with cerebral palsy. Dev Med Child Neurol 42: 724 - 7.

28. Van Der Linden Ml et al. (2003) Electrical stimulation of G Max in Children with CP; Effects on gait charecteristics and muscle strength. Dev Med Child Neurol 45: 385 - 90.

29. Fiky AE (2012) Efficacy of Surface Electrical Stimulation in treatment of cerebral Palsy Children: A Systemic Review. Cairo University. 17(1).

30. Vaz DV (2008) Effects of strength training aided by electrical stimulation on wrist muscle characteristics and hand function of children with hemiplegic cerebral palsy. Phys Occup Ther Pediatr. 28(4): 309 - 25.

31. Baker LL, yeh C, Wilson D, Watters RL (1979) Electrical stimulation for wrist and fingers for hemiplegic patients, Phys Ther. 59: 1495 - 1499.

32. Kamper Dget al. (2006) Effect of NMES for treatment of cerebral palsy on potential impairment mechanism: a pilot study, pediatric physiotherapy 18: $31-8$. 